Benchmarks

\title{
LUEGO: a cost and time saving gel shift procedure
}

\begin{abstract}
Nicolas Jullien and Jean-Paul Herman
Centre de Recherche en Neurobiologie et Neurophysiologie de Marseille (CRN2M), Centre National de la Recherche Scientifique (CNRS), and Faculté de Médecine Nord, Aix-Marseille Université, Marseille, France
\end{abstract}

BioTechniques 51:267-269 (October 2011) doi 10.2144/000113751

Keywords: electrophoretic mobility shift assay (EMSA); gel shift

Supplementary material for this article is available at www.BioTechniques.com/article/113751

We developed a new approach to prepare DNA probes for electrophoretic mobility gel shift assays that presents a number of advantages compared with the classical approach. The method relies on two complementary oligonucleotides containing the desired transcription factor binding sequence, one of them being extended at its $3^{\prime}$ end with a universal tail that complements a third, short oligonucleotide labeled with a fluorophore or any other label. The use of this third "universal" oligonucleotide allows labeling many different probes with minimal time, effort and cost. We show that probes prepared this way are as effective and reliable as probes prepared by conventional methods. We refer to this short oligonucleotide as LUEGO for labeled universal electrophoretic gel shift oligonucleotide.

The electrophoretic mobility shift assay (EMSA), also known as gel shift assay, is a popular and efficient technique to identify and analyze proteins binding to nucleic acids and to study different aspects of DNA-protein interactions. The principle of the technique is to set up a reaction between a probe - a short labeled double stranded DNA (although RNA or single stranded DNA can also be used) - and a protein, either purified or in a cellular/nuclear extract. The complex can be identified and quantified after separation from the unlabeled probe by nondenaturing electrophoresis (1-3).

One critical aspect of this assay is the labeling of the probe: there should exist a high sensitivity method to detect the label and the presence of the latter should not modify the formation and characteristics of the complex. Historically the first method to be used, and the gold standard to this day, is ${ }^{32} \mathrm{P} 5^{\prime}$-end labeling. However, the radioactive nature of the label creates issues related to regulatory framework, safety, cost, and the need for specific infrastructure. Moreover, the short half-life of ${ }^{32} \mathrm{P}$ requires that a given batch of labeled probe be used within a short time or that the labeling procedure be repeated with fresh radiolabel week after week, leading to increased costs and the use of different batches of probe for repeats of the experiment. Given these drawbacks, alternatives to radioactive labeling have been developed in recent years. One is the use of digoxigenin (DIG) or biotin label. While ensuring sensitivity comparable to that obtained with radioactive label, this approach requires additional post-electrophoresis steps (blotting, incubation with antibodies, chemiluminescent reaction), relies on an enzymatic reaction for visualization, and lacks the linearity of radioactive label $(4,5)$. Fluorescent labels, on the other hand, maintain a linear response curve and are the simplest to use, requiring only the direct scanning of the gel, although they are less sensitive than other labels (6-8). Common disadvantages of both of these non-radioactive labeling approaches are the relatively high cost when one has to use several different probes and the potentially long delay in obtaining the labeled probes from manufacturers.
To circumvent these last two problems of using non-radioactive probes, we propose here an alternative to classical DNA probes. The method relies on the use of two complementary oligonucleotides containing the protein binding sequence of interest, with one of them being extended at its $3^{\prime}$ end with a universal (i.e., identical for all probes) tail complementing a third, short oligonucleotide labeled with biotin or a fluorophore (Figure 1A). This third "universal" oligonucleotide allows the use of the same pre-prepared non-radioactive label for any specific probes used in a laboratory, with minimal time, effort and cost. Indeed, once this labeled short oligonucleotide has been obtained even with small-scale synthesis, quantities enough for thousands of individual reactions can be obtained relatively cheaply and stored for years - one needs to order only two inexpensive and quickly delivered unlabeled oligonucleotides containing the binding site of interest, with one of them having the $3^{\prime}$ extension complementary to the short oligonucleotide, and the whole probe can then be set up in minutes by annealing in solution. We call this short oligonucleotide LUEGO for labeled universal electrophoretic gel shift oligonucleotide and show here that its use leads to results that are identical with those obtained with traditional probes.

To avoid the introduction of artifacts, LUEGO has to satisfy several requirements: (i) the additional sequence introduced should not lead to unwanted binding of transcription factors; (ii) its melting temperature should be compatible with the gel shift procedure, in particular the electrophoresis step; and (iii) annealing with its complementary strand should be efficient. Based on these criteria, we selected the sequence 5'-GTGCCCTGGTCTGG-3', which is derived from a mouse SINE B1 sequence. Its short length limits non-specific binding, and it displays no homology to any known transcription factor binding sites, as shown by the Genomatix MatInspector software (9), which found no matches against Matrix Family Library version 8.3 vertebrate section. Its relatively high $\mathrm{G}-\mathrm{C}$ content $(71 \%)$ ensures a $\mathrm{T}$ high enough $\left(49.7^{\circ} \mathrm{C}\right.$, as calculated using the AmplifX software [http://ifrjr.nord.univ-mrs.fr/ AmplifX-Home-page]) to avoid melting of the oligonucleotide during the gel shift process, and it doesn't form self-dimers.

An important prerequisite for the use of the LUEGO-based probe is that assembly of the complete probe should be efficient and reproducible. We have previously observed that for classical probes made with two complementary 25 mer oligonucleotides, annealing efficiency in standard conditions is variable and sequence-dependent. However, a 5 -fold excess of the non-labeled strand is suffi- 
cient to anneal most or all of the labeled strand. Moreover, even a 50-fold excess of the unlabelled strand does not change the profile of gel shift assays for different probes (data not shown). For LUEGObased probes that require the annealing of three oligonucleotides, partial annealing can be an important issue as some partial combinations lacking LUEGO can bind the protein and so must be avoided. We showed that a 5:5:1 [LUEGO (red): short upper strand (green): long bottom strand (black); see Figure 1A] combination gives good results and (i) limits the green/black species that must be avoided because it would compete with the complete probe but not be detected and (ii) maximizes the formation of the complete (red/green/black) probe (Figure 1, B and C).

To demonstrate the usefulness and validity of this new system for EMSA, we have chosen two different DNA-protein complexes, in two standard situations. The first is based on the use of purified PIT-1 (POU1F1) protein and the 3P binding site from the rat prolactin promoter (10). A $6 \times \mathrm{His} \mathrm{N}$-tagged PIT-1 protein was produced in bacteria, purified ( $~ 90 \%$ purity), and used in comparative gel shift assays with different probes: a classic biotinylated probe and three LUEGO-based probes, where the LUEGO was labeled with biotin or IRDye700 at the $5^{\prime}$ end or double-labeled with $\mathrm{Cy} 5$ at the $5^{\prime}$ and $3^{\prime}$ ends. Comparison of gel shifts demonstrates that qualitatively identical results are obtained using these different probes (Figure 2A).As previously described (10,13), higher concentrations of the protein favor the formation of complexes containing dimer of the protein over complexes containing a single copy (Figure 2A, arrows), and this pattern is similarly observable with all the probes used."As previously described (10-11), higher concentrations of the protein favor the formation of complexes

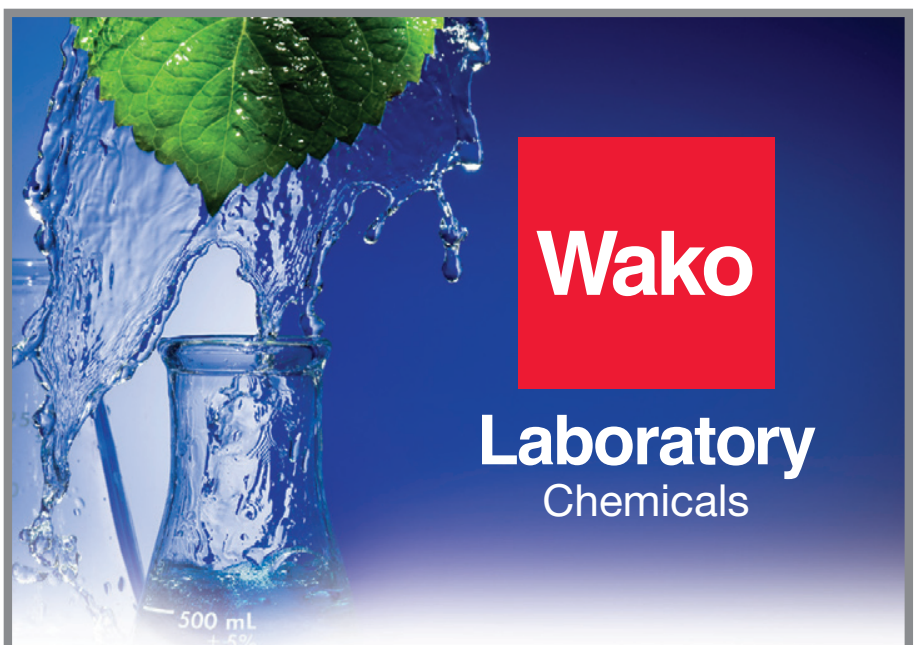

Your supplier of:

\section{-Anti Asialo GM1 -Lysyl Endopeptidase -Anti lba-1 -Bambanker}

\section{Visit our \\ Online Catalog to see our other quality products}

A

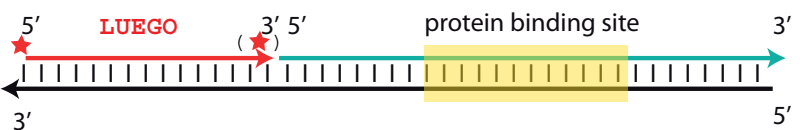

B

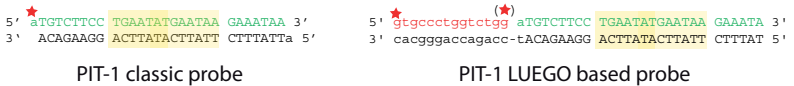

C

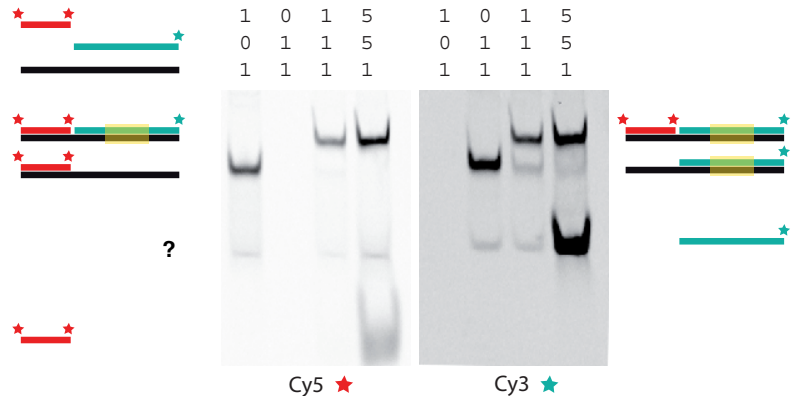

Figure 1. Principle and annealing conditions for LUEGO-based probes. (A) General scheme of LUEGO-based probes. The LUEGO strand is represented in red, the specific probe strand in green, with the specific protein binding site highlighted with yellow. The red stars represent the labeled nucleotides, with the one in parentheses indicating the option for doubling the label that we used for the $2 \times$ Cy5 probe. (B) Application of the LUEGO strategy to the PIT-1 (POU1F1) probe. Color coding of nucleotides is the same as for panel A. (C) Annealing efficiency of the PIT-1 LUEGO-based probe (2xCy5labeled, red stars). Oligonucleotides are mixed at the ratios indicated ("1" corresponding to $5 \mu \mathrm{M}$ ) and processed as in a real gel shift assay except that no protein is added and the gel is $12 \%$ acrylamide. To detect all partial combinations, the specific oligo (green) has been labeled at its $3^{\prime}$ end with Cy3 (green star). The question mark indicates a band that migrates at almost the same position as the green oligonucleotide. It is thought to be a nonlinear form of the red/black annealed oligonucleotides. For technical details, see the Supplementary Materials and methods.

containing dimer of the protein over complexes containing a single copy (Figure $2 \mathrm{~A}$, arrows), and this pattern is similarly observable with all the probes used. A more complete titration experiment shows that the the doubly labeled Cy5-LUEGO-based probe gives the same quantitative results as the classic probe (Figure 2B). It should be stressed that the total signal intensity (sum of the intensities measured in the different bands of a given lane) is very homogeneous ( $<5 \%$ variation) between lanes (data not shown), indicating that binding of the protein to the probe does not change the brightness of the two Cy5 fluorophores in the doubly labeled Cy5-LUEGO probe.

The second system used was that of binding of AP-1 in a complex mixture to its consensus binding sequence, a widely used and well characterized system (12-13). Nuclear extracts were prepared from control GH4C1 cells or $\mathrm{GH} 4 \mathrm{Cl}$ cells exposed to forskolin to induce the formation of the AP-1 complex. The extracts were incubated with either the biotinylated classic probe or the LUEGO- $2 \times \mathrm{Cy} 5$-based probe. Again, the two systems gave equivalent results, showing the presence in the extract of a factor binding the probe in a specific manner, as indicated by the competition for binding by unlabelled probe but not by mutated probe. This factor contains FRA-2 as demonstrated by the supershifted band in presence of an anti-FRA2 antibody (Figure 2C).

In conclusion, LUEGO-based probes compare favorably with classical non-radioactive probes for EMSA and, in fact, give identical results. The approach presents several practical advantages to classical probes. The first is cost, as biotin- or fluorophore-labeled oligonucleotides are 5 to 20 times more expensive than non-labeled oligonucleotides, and when several probes are needed, the cost of the probes can 

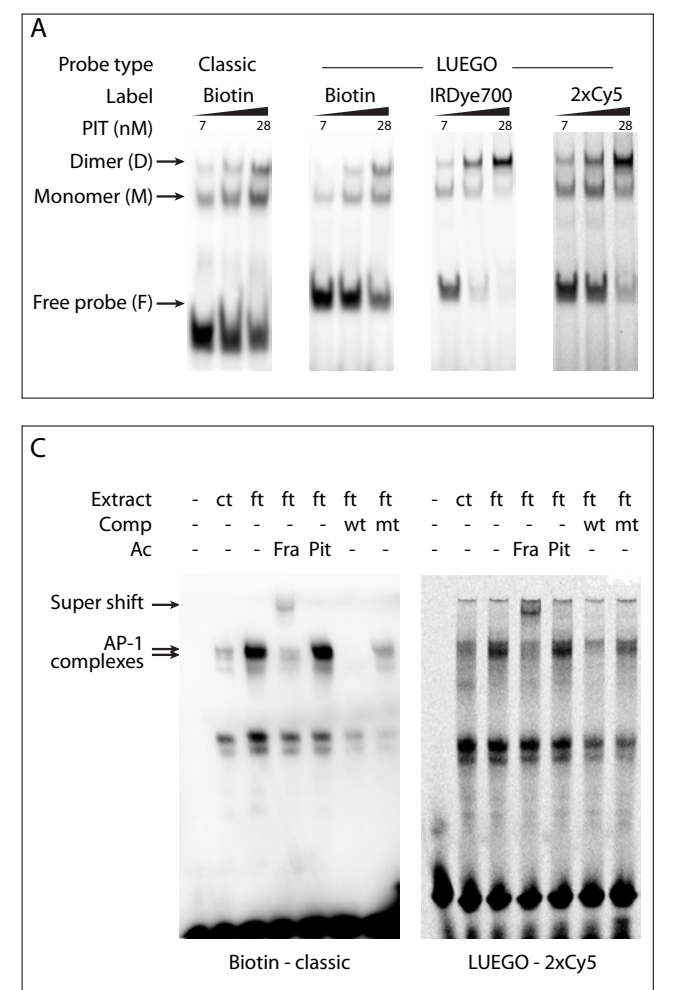

Figure 2. Comparison of classical and LUEGO-based probes in two applications. (A) Comparison of gel shift experiments done using classic biotin-labeled or different LUEGO-based probes and purified PIT-1 protein. (B) Gel shift titrations comparing the classic PIT-1 probe labeled with a single Cy5 and a LUEGO-based PIT-1 probe labeled with $2 \times C y 5$. The two gels were scanned using the same parameters and the images were set with the same display range (192 to 22805 gray levels). The plot shows the results of quantification using ImageJ, the ordinate corresponding to the intensity of the bound probe (sum of the intensities of the monomer and dimer bands) relative to that of the total (bound plus free) probe. Note again the progressive shift toward PIT-1 dimer containing complexes with higher protein concentrations. The titration curves obtained with the two systems are strictly superimposable, as shown on the bottom graph. (C) Comparison of gel shift experiments done using classic biotinlabeled or doubly labeled Cy5-LUEGO-based probes for AP-1 and nuclear extracts from control (ct) or forskolin-treated (ft) GH4C1 cells. The formation of the labeled complex could be competed out (comp) in both cases with the wild-type (wt), but not with the mutated (mt), unlabeled probe. AP-1 complex was supershifted with an antibody directed against FRA-2 (Fra) but not against PIT-1 (Pit) in both cases. For technical details, see the Supplementary Materials and methods.

become a significant factor. With LUEGObased probes, one has to obtain the labeled oligonucleotide (i.e., LUEGO) only once, and the probe will last for months or years (we have observed no reduction in efficiency over more than 6 months and many freeze/thaw cycles). Creating new probes then involves only ordering inexpensive non-labeled oligonucleotides that are delivered significantly faster than labeled ones. The ability to generate a collection of LUEGOs with different labels (different fluorophores, biotin, etc) can, moreover, permit new developments in EMSA such as direct comparison in a single lane of binding to two or more different probes. An additional advantage is the possibility to attach multiple fluorophores to the LUEGO, thus increasing the sensitivity of detection of the DNA-protein complex and making fluorescence an alternative of choice to biotin-based detection.

\section{Acknowledgments}

We thank Eric Cortegianni from Integrated DNA Technologies for providing IRDye and Cy oligonucleotide at the best prices. We thank Michel Khrestchatisky for access to the Typhoon scanner and Pierre Luciano for access to the Odyssey scanner.

\section{Competing interests}

The authors declare no competing interests.

\section{References}

1. Lane, D., P. Prentki, and M. Chandler. 1992. Use of gel retardation to analyze protein-nucleic acid interactions. Microbiol. Rev. 56:509-528.

2. Laniel, M.A., A. Béliveau, and S.L. Guérin. 2001. Electrophoretic mobility shift assays for the analysis of DNA-protein interactions. Methods Mol. Biol. 148:13-30.
3. Hellman, L.M. and M.G. Fried. 2007. Electrophoretic mobility shift assay (EMSA) for detecting protein-nucleic acid interactions. Nat. Protocols 2:1849-1861.

4. Heidebrecht, F., A. Heidebrecht, I. Schulz, S.E. Behrens, and A. Bader. 2009. Improved semiquantitative Western blot technique with increased quantification range. J. Immunol. Methods 345:40-48.

5. Höltke, H.J., G. Sagner, C. Kessler, and G. Schmitz. 1992. Sensitive chemiluminescent detection of digoxigenin-labled nucleic acids: a fast and simple protocol and its applications. Biotechniques 12:104-113.

6. Ruscher, K., M. Reuter, D. Kupper, G. Trendelenburg, U. Dinargl, and A. Meisel. 2000. A fluorescence based non-radioactive electrophoretic mobility shift assay. J. Biotechnol. 78:163-170.

7. Ying, B.-W., D. Fourmy, and S. Yoshizawa. 2007. Substitution of the use of radioactivity by fluorescence for biochemical studies of RNA. RNA 13:2042-2050.

8. Anderson, B.J., C. Larkin, K. Guja, and J.F. Schilbach. 2008. Using fluorophore-labeled oligonucleotides to measure affinities of protein-DNA interactions. Methods Enzymol. 450:253-272.

9. Cartharius, K., K. Frech, K. Grote, B. Klocke, M. Haltmeier, A. Klingenhoff, M. Frisch, M. Bayerlein, and T. Werner. 2005. MatInspector and beyond: promoter analysis based on transcription factor binding sites. Bioinformatics 21:2933-2942.

10. Howard, P.W. and R.A. Maurer. 1995. A composite Ets/Pit-1 binding site in the prolactin gene can mediate transcriptional responses to multiple signal transduction pathways. J. Biol. Chem. 270:20930-20936.

11. Voss, J.W., L. Wilson, and M.G. Rosenfeld. 1991. POU-domain proteins Pit-1 and Oct-1 interact to form a heteromeric complex and can cooperate to induce expression of the prolactin promoter. Genes Dev. 5:1309-1320.

12. Baler, R. and D.C. Klein. 1995. Circadian expression of transcription factor Fra-2 in the rat pineal gland. J. Biol. Chem. 270:27319-27325.

13. Sharma, S.C. and J.S. Richards. 2000. Regulation of AP1 (Jun/Fos) factor expression and activation in ovarian granulosa cells. Relation of JunD and Fra2 to terminal differentiation. J. Biol. Chem. 275:33718-33728.

Received 29 June 2011; accepted 8 September 2011.

Address correspondence to Faculté de Médecine Nord, Aix-Marseille Université. CS80011, Bd Pierre Dramard, 13344 Marseille Cedex 15, France. e-mail: nicolas.jullien@univmed.fr

To purchase reprints of this article, contact: biotechniques@fosterprinting.com 